\title{
V-BLAST Without Optimal Ordering: Analytical Performance Evaluation for Rayleigh Fading Channels
}

\author{
Sergey Loyka, Senior Member, IEEE, and Francois Gagnon, Senior Member, IEEE
}

\begin{abstract}
The Bell Labs layered space-time (BLAST) algorithm is simple, and hence, a popular choice for a multiple-input multiple-output (MIMO) receiver. Its bit-error rate (BER) performance has been studied mainly using numerical (Monte Carlo) techniques, since exact analytical evaluation presents serious difficulties. Close examination of the problem of BLAST BER performance analysis reveals that the major difficulty for analytical evaluation is due to the optimal ordering procedure. Hence, we analyze the algorithm performance without optimal ordering. While this is a disadvantage of the analysis, there are certain advantages as well. Exact closed-form analytical evaluation is possible for arbitrary number of transmit and receive antennas in an independent, identically distributed Rayleigh fading channel, which provides deep insight and understanding that cannot be gained using the Monte Carlo approach alone. A result on the maximum ratio combining weights, which is used at each detection step, is derived to obtain a number of results: independence of noise, distribution of signal-to-noise ratio (SNR), and block- or bit-error rates. We present a detailed analysis and expressions for uncoded error rates at each detection step, which hold true for any modulation format and take simple closed form in some cases. Asymptotic form of these expressions for large SNRs is particularly simple. Extensive Monte Carlo simulations validate the analytical results and conclusions.
\end{abstract}

Index Terms-Bit-error rate (BER), Bell Labs layered space-time (BLAST), error propagation, multiantenna systems, multiple-input multiple-output (MIMO), performance analysis.

\section{INTRODUCTION}

$\mathbf{T}$ HE Bell Labs layered space-time (BLAST) algorithm is simple and hence a popular choice for a multiple-input multiple-output (MIMO) receiver, which also achieves a significant portion of, or, under certain conditions, the full MIMO capacity [1]-[3]. Its bit-error rate (BER) performance has been studied mainly numerically (Monte Carlo (MC) techniques) since analytical evaluation presents serious difficulties, especially when no bounds or approximations are used. While the

Paper approved by A. Lozano, the Editor for Wireless Communication of the IEEE Communications Society. Manuscript received November 25, 2004; revised June 22, 2005 and November 7, 2005. This work was supported in part by the Natural Sciences and Engineering Research Council of Canada. This paper was presented in part at the IEEE Vehicular Technology Conference, Los Angeles, CA, September 2004.

S. Loyka is with the School of Information Technology and Engineering (SITE), University of Ottawa, Ottawa, ON K1N 6N5, Canada (e-mail: sergey. loyka@ieee.org).

F. Gagnon is with the Department of Electrical Engineering, Ecole de Technologie Superieure, Montreal, QC H3C 1K3, Canada (e-mail: francois.gagnon@etsmtl.ca).

Digital Object Identifier 10.1109/TCOMM.2006.876875 performance of $2 \times n$ system (i.e., with two transmit (Tx) and $n$ receive ( $\mathrm{Rx}$ ) antennas) can be analytically evaluated in a closed form without using any bounds [4], [5], an extension of the analysis to the general case of system has not been found yet. Hence, various bounds and approximations have been employed to attack the problem [6]. Consequently, the solutions found are limited in some way. An additional limitation of the analysis in [4] and [5] is that a noncoherent (i.e., powerwise) equal gain combining (NC EGC) was used after interference nulling out (projection), which is not optimum, and that the after-projection noise correlation was ignored.

The approach adopted in this paper is different. Close examination of the problem of BLAST BER performance analysis reveals that the major difficulty for closed-from exact analytical evaluation is due to the optimal ordering procedure. Hence, we analyze the algorithm performance without optimal ordering. Clearly, this is a disadvantage of the analysis. However, there are certain advantages as well: 1) closed-form exact analytical evaluation is possible in the general case of system, which results in exact BER expressions; 2) this provides deep insight and understanding that cannot be gained using the MC approach alone; 3) there exists a hope that the techniques developed can be further extended to account for the optimal ordering; 4) comparing the performance of the no-ordering algorithm with that with the optimal ordering allows one to better understand the advantages provided by the ordering and various differences in the performance; and 5) computational complexity of the algorithm without optimal ordering is significantly less and, hence, an implementation complexity is smaller. Contrary to [5], we employ the optimum maximum ratio combining (MRC) after the interference projection (taking into account the after-projection noise correlation), which provides the best performance in terms of the output signal-to-noise ratio (SNR), and hence, the BER. This also results in exact closed-form expressions for unconditional average BER at each step, which are not available in [5]. Thus, a precise quantification of the error propagation effect is possible.

The approach consists essentially of obtaining new results on the optimal MRC weights without ordering. These weights are expressed as a product of a projection matrix by part of the channel matrix and it is thus possible to prove that the after-combining noise components at each detection step are Gaussian and independent of each other. A closed-form expression of the output SNR distributions at each step is obtained with an assumption of error-free detection at the previous steps. These distributions are used to obtain relatively precise outage, block-, 
bit-, and total bit-error rates. A more detailed analysis, which still makes good use of the MRC weight properties but with conditioning on previous step error events, permits obtaining exact BER expressions for binary phase-shift keying (BPSK) modulation without the error-free detection assumption, i.e., taking into account the error propagation. Finally, we point out that the uncoded diagonal (D)-BLAST performance is identical to that of vertical (V)-BLAST in terms of the total error rate, and that all the results also apply to the QR-decomposition-based V-BLAST.

It should be mentioned that there exists certain similarity between the V-BLAST, the decision-feedback multiuser detector (DF-MUD) [11], [16] and the decision-feedback equalizer (DFE) [10], [15] as they employ essentially equivalent signal processing algorithms to eliminate interference from different streams, users, or symbols ([11] gives a detailed discussion of various MUD algorithms and their performance analysis; it also provides an extensive reference list). However, the performance of these algorithms may differ significantly, especially in a fading channel (for example, while adding more receive antennas in the V-BLAST increases the diversity order, adding more spreading sequences in a multiuser code-division multiple-access (CDMA) system with the DF-MUD or more taps in the DFE does not). One way to obtain the average error rates for the V-BLAST would be to employ, with proper modifications, the instantaneous error rate results obtained for the DF-MUD [11], [16], [17] or the DFE [18] (note that these results often rely on various bounds, approximations, or simulations, especially when taking into account the error propagation), and to average them out over the channel statistics (fading). In this paper, we adopt a more direct and also simpler approach of step-by-step analysis of the V-BLAST and introduce "semi-instantaneous" error rates, which rely on the fact that the interstream interference (ISTI) (due to the error propagation) is Gaussian in a Rayleigh fading channel. This simplifies the derivation of average error rates significantly and results in exact closed-form expressions, which take into account the error propagation without any approximations or bounds. As usually, high-SNR approximations of those expressions offer significant insight into the system performance.

The paper is organized as follows. Section II presents basic channel and V-BLAST algorithm models and the assumptions made. Section III gives a detailed analysis of the after-combining instantaneous SNR and noise at each step. Section IV gives a generic analysis of the outage probability, instantaneous and average BER, block error rate (BLER), and total bit-error rate (TBER). A relationship between BLER and TBER is also discussed. Section V specifies the results of Section IV to the case of $2 \times 2,2 \times n$, and $m \times n$ systems with BPSK modulation and gives exact closed-form BER expressions. Section VI provides MC simulation results, which validate the analytical evaluation of the previous sections. Finally, Section VII concludes the paper.

\section{THE V-BLAST ALGORITHM}

The main idea of the BLAST architecture is to split the information bit stream into several substreams and transmit them in parallel using a set of Tx antennas (the number of Tx antennas equals the number of substreams) at the same time and frequency. At the Rx end, each Rx antenna "sees" all the transmitted signals, which are mixed due to the nature of the wireless propagation channel. Using appropriate signal processing at the receiver, these signals can be separated so that the matrix wireless channel is transformed into a set of virtual parallel independent channels (provided that the multipath is rich enough).

The standard baseband system model is given by

$$
\mathbf{r}=\mathbf{H s}+\boldsymbol{\xi}
$$

where $\mathbf{s}$ and $\mathbf{r}$ are the Tx and Rx vectors, respectively, $\mathbf{H}$ is the $n \times m$ channel matrix, i.e., the matrix of the complex channel gains between each Tx and each Rx antenna, $n$ is the number of Rx antennas, $m$ is the number of Tx antennas, $n \geq m$, and $\boldsymbol{\xi}$ is the additive white Gaussian noise (AWGN), which is assumed to be $\mathcal{C N}\left(\mathbf{0}, \sigma_{0}^{2} \mathbf{I}\right)$, i.e., independent and identically distributed (i.i.d.) in each branch.

The job of the V-BLAST algorithm is to find $\mathbf{s}$ given $\mathbf{r}$ and $\mathbf{H}$ in a computationally efficient way. The V-BLAST processing begins with the first Tx symbol and proceeds in sequence to the $m$ th symbol. When the optimal ordering procedure is employed, the Tx indexing is changed prior to the processing. The main steps of the algorithm are as follows [1], [3].

1) The interference cancellation step: At the $i$ th processing step (i.e., when the signal from the $i$ th transmitter is detected) the interference from the first $(i-1)$ transmitters can be subtracted based on the estimations of the Tx symbols and the knowledge of the channel matrix $\mathbf{H}$

$$
\mathbf{r}_{i}^{\prime}=\mathbf{r}-\sum_{j=1}^{i-1} \mathbf{h}_{j} \hat{s}_{j}
$$

where $\mathbf{h}_{j}$ is the $j$ th column of $\mathbf{H}$, and $\hat{s}_{j}$ are the detected symbols (which are assumed to be error-free).

2) The interference nulling step: Based on the knowledge of the channel matrix, the interference from yet-to-be-detected symbols can be nulled out using the Gram-Schmidt orthogonalization process (applied to the column vectors of $\mathbf{H}$ ) and orthogonal projection on the subspaced spanned by yet-to-be-detected symbols

$$
\mathbf{r}_{i}^{\prime \prime}=\mathbf{P}_{i} \mathbf{r}_{i}^{\prime}
$$

where $\mathbf{P}_{i}$ is the projection matrix on the subspace orthogonal to that spanned by $\left\{\mathbf{h}_{i+1} \mathbf{h}_{i+2}, \ldots, \mathbf{h}_{m}\right\}: \mathbf{P}_{i}=\mathbf{I}-$ $\mathbf{H}_{i}\left(\mathbf{H}_{i}^{+} \mathbf{H}_{i}\right)^{-1} \mathbf{H}_{i}^{+}$, where $\mathbf{H}_{i}=\left[\mathbf{h}_{i+1} \mathbf{h}_{i+2}, \ldots, \mathbf{h}_{m}\right]$, [8] and "+" means Hermitian conjugate. Clearly, this corresponds to complete [i.e., zero-forcing (ZF)] ISTI cancellation. While the minimum mean-square error (MMSE) algorithm would perform better, it is more difficult to implement, and also is much more challenging to analyze. We further assume the ZF ISTI cancellation.

3) The optimal ordering procedure: The order of symbol processing is organized according to their after-processing 
SNRs in the decreasing order, i.e., the symbol with highest SNR is detected first.

\section{ANALYSIS OF THE V-BLAST ALGORITHM}

The following basic assumptions are employed in this paper:

1) the channel is random, quasi-static (i.e., fixed for every frame of information bits but varying from frame to frame), frequency-independent (i.e., negligible delay spread); the components of $\mathbf{H}$ are $\mathcal{C N}(0, \mathbf{I})$ (i.e., i.i.d. Rayleigh fading with unit average power gain);

2) equal-power constellations are used;

3) the Tx signals, noise, and channel gains are independent of each other;

4) perfect channel knowledge is available at the receiver, but not at the transmitter;

5) there is no performance degradation due to synchronization and timing errors;

6) no coding is used.

It should be noted that some of the results below do not need all these assumptions. As it was indicated above, the optimal ordering procedure will be omitted in this paper. We follow the approach to V-BLAST analysis proposed in [4] and [5], where it was shown that the conditional (i.e., assuming no detection error at the first $(i-1)$ steps) after-processing instantaneous (i.e., for given channel instant) signal power $P_{s i}$ at the $i$ th processing step is

$$
P_{s i} \sim \chi_{2(n-m+i)}^{2}
$$

where $\sim$ means equal in distribution, and different $P_{s i}$ are independent of each other (this follows directly from the geometric analysis in [5] and also from the QR decomposition of $\mathbf{H}$; see the end of Section $\mathrm{V}$ for details). The $i$ th step has diversity order equal to $(n-m+i)$, the smallest one being at the first step, and the largest at the last one. Note that the fact that the distribution is conditional (no error propagation) does not limit the analysis, since, as we show below, the conditional distribution at each step is sufficient to find the BLER and outage probability taking into account the error propagation. The distribution of $P_{s i}$ follows also from the Bartlett decomposition of the complex Wishart matrix [7] (see the end of Section V).

The best way to improve the output SNR is to use MRC after the interference nulling-out step, i.e., to form the decision variable

$$
\widehat{r}_{i}=\mathbf{w}_{i}^{+} \mathbf{r}_{i}^{\prime \prime}
$$

where $\mathbf{w}_{i}^{+}$are the optimum weights. However, the well-known MRC expressions for the weights and the output SNR cannot be applied directly, since the orthogonal projection during the nulling-out step [see (3)] results in correlated branch noise. The after-projection noise correlation matrix is

$$
\mathbf{C}_{\xi i}=\left\langle\mathbf{P}_{i} \xi \xi^{+} \mathbf{P}_{i}\right\rangle=\sigma_{0}^{2} \mathbf{P}_{i}
$$

where \langle\rangle denotes expectation (in this case, over the noise). The MRC combining weights $\mathbf{w}_{i}$ are given in this case by the solution of the following generalized eigenvalue problem (see Appendix A):

$$
\left(\mathbf{C}_{s i}-\gamma_{i} \mathbf{C}_{\xi i}\right) \mathbf{w}_{i}=\mathbf{0}
$$

where $\mathbf{C}_{s i}$ is the after-projection signal covariance matrix at step $i$. Remarkably, as the detailed analysis of Appendix A demonstrates, the output SNR is still the same as that for i.i.d. branch noise

$$
\gamma_{i}=\frac{P_{s i}}{\sigma_{0}^{2}}=\frac{\mathbf{y}_{i}^{+} \mathbf{y}_{i}}{\sigma_{0}^{2}}=\frac{\mathbf{h}_{i}^{+} \mathbf{P}_{i} \mathbf{h}_{i}}{\sigma_{0}^{2}}
$$

where $\mathbf{y}_{i}=\mathbf{P}_{i} \mathbf{h}_{i}$ is the after-projection signal, and we have assumed that $\left|s_{i}\right|^{2}=1$ (i.e., an equal-power constellation. The results below can also be generalized to an unequal-power case). We stress that this is a nontrivial result that holds true because of special structure of the projection matrices $\mathbf{P}_{i}$. The optimum (MRC) weights are given by

$$
\mathbf{w}_{i}=\mathbf{P}_{i} \mathbf{h}_{i}
$$

Since these weights already include the projection, they can be applied directly to $\mathbf{r}_{i}^{\prime}$. The output signal can be presented as

$$
\begin{aligned}
\widehat{r_{i}} & =\mathbf{w}_{i}^{+} \mathbf{r}_{i}^{\prime}=r_{s i}+r_{\xi i} \\
r_{s i} & =\mathbf{w}_{i}^{+} \mathbf{h}_{i} s_{i} \\
r_{\xi i} & =\mathbf{w}_{i}^{+} \boldsymbol{\xi} .
\end{aligned}
$$

Since $P_{s i}$ at different steps are independent of each other, so are $\gamma_{i}$. From this, however, it does not follow that the decisions and, hence, errors at each step are independent of each other, as the latter requires for the noise $r_{\xi i}$ to be independent at each step, not just the SNR. To demonstrate that the instantaneous noise is indeed independent at each step, we first show that

$$
\mathbf{P}_{i} \mathbf{P}_{j}=\mathbf{P}_{i} \quad \forall j>i .
$$

Indeed, $\mathbf{P}_{j}$ can be presented as

$$
\mathbf{P}_{j}=\mathbf{I}-\sum_{k=j+1}^{m} \mathbf{v}_{k} \mathbf{v}_{k}^{+}=\mathbf{I}-\sum_{k=j+1}^{m} a_{k j} \mathbf{h}_{k} \mathbf{h}_{k}^{+}
$$

where orthonormal vectors $\left\{\mathbf{v}_{j+1}, \mathbf{v}_{j+2} \ldots \mathbf{v}_{m}\right\}$ satisfy the following requirement:

$$
\operatorname{span}\left\{\mathbf{v}_{j+1}, \mathbf{v}_{j+2}, \ldots, \mathbf{v}_{m}\right\}=\operatorname{span}\left\{\mathbf{h}_{j+1}, \mathbf{h}_{j+2} \ldots \mathbf{h}_{m}\right\}
$$

The second equality in (12) follows from the Gram-Schmidt orthonormalization procedure [5]. Since

$$
\mathbf{P}_{i} \mathbf{h}_{j}=\mathbf{0} \quad \forall j>i
$$


it follows that

$$
\mathbf{P}_{i} \mathbf{P}_{j}=\mathbf{P}_{i}-\sum_{k=j+1}^{m} a_{k j} \mathbf{P}_{i} \mathbf{h}_{k} \mathbf{h}_{k}^{+}=\mathbf{P}_{i} \quad \forall j>i
$$

which is what one would expect intuitively as $\mathbf{P}_{i}$ eliminates all the components in span $\left\{\mathbf{h}_{i+1}, \mathbf{h}_{i+2}, \ldots, \mathbf{h}_{m}\right\}$, which includes also the components in $\operatorname{span}\left\{\mathbf{h}_{j+1}, \mathbf{h}_{j+2}, \ldots, \mathbf{h}_{m}\right\}$ for $j>i$ and, hence, $\mathbf{P}_{j}$ is redundant. Using this result, one can show that

$$
\mathbf{w}_{i}^{+} \mathbf{w}_{j}=\mathbf{h}_{i}^{+} \mathbf{P}_{i} \mathbf{P}_{j} \mathbf{h}_{j}=\mathbf{0} \quad \forall i \neq j
$$

i.e., optimum weights at different steps are orthogonal to each other.

Consider now the output noise covariance matrix

$$
R_{i j}=\left\langle r_{\xi i} r_{\xi j}\right\rangle
$$

where the expectation is over the noise. Using (9), (10), and (16), one obtains

$$
R_{i j}=\sigma_{0}^{2} \mathbf{w}_{i}^{+} \mathbf{w}_{j}=\sigma_{0}^{2}\left|\mathbf{w}_{i}\right|^{2} \delta_{i j}
$$

where $\delta_{i j}=1$ if $i=j$ and 0 otherwise, and $\left|\mathbf{w}_{i}\right|=\sqrt{\mathbf{w}_{i}^{+} \mathbf{w}_{i}}$ denotes the $L_{2}$ (Euclidean) norm. Since $r_{\xi i}=\mathbf{w}_{i}^{+} \boldsymbol{\xi}$ are complex Gaussian (for given channel realization), independence follows from zero correlation. Hence, the decisions and errors are independent at each step, and the conditional error rates are independent, too. This result parallels the one for ZF DF-MUD [11], as they employ similar processing algorithms. ${ }^{1}$

\section{OUtAgE PROBABILITY AND ERROR RATES}

As (8) demonstrates, statistical analysis of the SNR for a fading channel is equivalent to that of the output signal power. Using the distribution of the signal power at each detection step given above (i.e., (4); see [5] for more details), the conditional outage probability at step $i$, conditioned on error-free detection in the previous steps, can be found immediately

$$
F_{i}(x)=\operatorname{Pr}\left[\gamma_{i} / \gamma_{0}<x\right]=1-e^{-x} \sum_{k=0}^{n-m+i-1} x^{k} / k !
$$

where $\gamma_{0}$ is the average per-branch SNR, and the corresponding probability density function (PDF) is

$$
f_{i}(x)=d F_{i}(x) / d x=x^{n-m+i-1} e^{-x} /(n-m+i-1) !
$$

Due to the independence of $\gamma_{i}$ at different steps, the joint SNR PDF is the product of the step SNR PDFs (20).

\footnotetext{
${ }^{1}$ This was pointed out by an anonymous reviewer.
}

\section{A. Error-Rate Evaluation}

The conditional (i.e., given no error at the first $(i-1)$ steps) average (over the channel statistics) BER at the $i$ th step can be expressed in the standard form

$$
\overline{P_{e, i}}=\gamma_{0}^{-1} \int_{0}^{\infty} P_{e}(\gamma) f_{i}\left(\gamma / \gamma_{0}\right) d \gamma
$$

where $P_{e}(\gamma)$ is the instantaneous BER (for given $\gamma$ ), which is determined by the modulation format, and $\bar{P}$ denotes an average error rate. Noting that $f_{i}$ has the $\chi_{2(n-m+i)}^{2}$ distribution, the average BER can be expressed in closed form for many modulation formats [10]. Since the instantaneous conditional BER at step $i$ is simply $P_{e}\left(\gamma_{i}\right)$, the probability of having an error at step $i$ and no errors at steps 1 to $(i-1)$ is

$$
P_{e}\left(\gamma_{i}\right) \prod_{k=1}^{i-1}\left(1-P_{e}\left(\gamma_{k}\right)\right)
$$

where we have taken into account the error independence. The probability of having at least one error at steps 1 to $i$ is

$$
\begin{aligned}
P_{i} & =1-\prod_{k=1}^{i}\left(1-P_{e}\left(\gamma_{k}\right)\right) \\
& =\sum_{j=1}^{i} P_{e}\left(\gamma_{j}\right) \prod_{k=1}^{j-1}\left(1-P_{e}\left(\gamma_{k}\right)\right) \\
& \leq \sum_{j=1}^{i} P_{e}\left(\gamma_{j}\right) .
\end{aligned}
$$

Finally, defining a BLER as a probability of having at least one error at the detected $\mathrm{Tx}$ vector, one can express it as

$$
\begin{aligned}
P_{B} & =P_{m} \\
& =1-\prod_{k=1}^{m}\left(1-P_{e}\left(\gamma_{k}\right)\right) \\
& =\sum_{j=1}^{m} P_{e}\left(\gamma_{j}\right) \prod_{k=1}^{j-1}\left(1-P_{e}\left(\gamma_{k}\right)\right) \\
& \leq \sum_{j=1}^{m} P_{e}\left(\gamma_{j}\right) .
\end{aligned}
$$

We emphasize that in order to find BLER, one needs only the conditional error probabilities at each step; the error propagation does not affect (24) as the BLER is independent of it. Another way to look at the probabilities in (23) and (24) is as the error probability at steps $i$ and $m$, respectively, assuming $100 \%$ error propagation (i.e., an error at any previous step necessarily results in an error in any further steps). Detailed analysis demonstrates that this is a somewhat exaggerated assumption; the actual error propagation is less than $100 \%$. Hence, these expressions provide 
TABLE I

ERROR RATES AND CORRESPONDING SYMBOLS

\begin{tabular}{|c|c|c|}
\hline Name & Symbol & $\begin{array}{c}\text { First } \\
\text { appearance }\end{array}$ \\
\hline Instantaneous error rate (for given SNR $\gamma$ ) & $P_{e}(\gamma)$ & Eq. 21 \\
\hline $\begin{array}{l}\text { The probability of having at least one error at } \\
\text { steps } 1 \text { to } i\end{array}$ & $P_{i}=1-\prod_{k=1}^{i}\left(1-P_{e}\left(\gamma_{k}\right)\right)$ & Eq. 23 \\
\hline $\begin{array}{l}\text { Conditional instantaneous error rate at step } i \\
\text { (the probability of having an error at step } i \text { given } \\
\text { no errors at steps } 1 \text { to }(i-1) \text { ) }\end{array}$ & $P_{e}\left(\gamma_{i}\right)$ & Eq. 22 \\
\hline $\begin{array}{l}\text { Unconditional instantaneous error rate at step } i \\
\text { (includes the error propagation from the previous } \\
\text { steps) }\end{array}$ & $P_{u i}$ & Eq. 25 \\
\hline $\begin{array}{l}\text { Instantaneous block error rate (BLER) } \\
\text { (probability of having at least one error at the } \\
\text { detected Tx vector) }\end{array}$ & $P_{B}=P_{m}$ & Eq. 24 \\
\hline $\begin{array}{l}\text { Instantaneous total bit error rate (TBER) (the } \\
\text { error rate at the single output steam, to which all } \\
\text { the sub-streams are merged) }\end{array}$ & $P_{e t}=\lim _{N \rightarrow \infty} \frac{N_{e}}{N}$ & Eq. 30 \\
\hline $\begin{array}{l}\text { Conditional average error rate at i-th step } \\
\text { (assuming no error at the previous steps) }\end{array}$ & $\begin{array}{l}\quad \overline{P_{e, i}}=\gamma_{0}^{-1} \int_{0}^{\infty} P_{e}(\gamma) f_{i}\left(\gamma / \gamma_{0}\right) d \gamma\left(\mathrm{BER}_{i},\right. \\
\text { no err. prop., on Fig. 3-4) }\end{array}$ & Eq. 21 \\
\hline $\begin{array}{l}\text { The average probability of having at least one } \\
\text { error at steps } 1 \text { to } i\end{array}$ & $\bar{P}_{i}=1-\prod_{k=1}^{i}\left(1-\bar{P}_{e}\left(\gamma_{k}\right)\right)$ & Eq. 27 \\
\hline Unconditional average error rate at step $i$ & $\bar{P}_{u i}\left(\mathrm{BER}_{i}\right.$ on Fig. $\left.1,3-6\right)$ & Eq. 27 \\
\hline Average BLER & $\bar{P}_{B}$ (BLER on Fig. 3-6) & Eq. 26 \\
\hline Average TBER & $\overline{P_{e t}}=\frac{1}{m} \sum_{i=1}^{m} \overline{P_{u i}}$ (TBER on Fig.1,3-6) & Eq. 33 \\
\hline Average BPSK BER with $n$-th order MRC & $\bar{P}_{(n)}^{M R C}$ & Eq. 45 \\
\hline
\end{tabular}

upper bounds on respective error probabilities. Consequently, the $i$ th step (unconditional) error probability $P_{u i}$ can be bounded as

$$
P_{e}\left(\gamma_{i}\right) \leq P_{u i} \leq P_{i}
$$

where the lower bound follows from the assumption of no error propagation at all. Using the independence of noise at different steps, and also the fact that $\gamma_{i}$ and $\gamma_{k}$ are independent for $i \neq k$ in i.i.d. Rayleigh fading channel, the average BLER is

$$
\bar{P}_{B}=\sum_{j=1}^{m} \bar{P}_{e, j} \prod_{k=1}^{j-1}\left(1-\bar{P}_{e, k}\right) \leq \sum_{j=1}^{m} \bar{P}_{e, j} .
$$

In a similar way, the average (unconditional) $\overline{P_{u i}}$ step error rate can be bounded as

$$
\overline{P_{e, i}} \leq \overline{P_{u i}} \leq \overline{P_{i}}=\sum_{j=1}^{i} \bar{P}_{e, j} \prod_{k=1}^{j-1}\left(1-\bar{P}_{e, k}\right) \leq \sum_{j=1}^{i} \bar{P}_{e, j}
$$

Let us now consider its asymptotic behavior for large average SNR, $\gamma_{0} \gg 1$. In this case, $\overline{P_{e, i}} \ll 1$, and the product term represents a second-order effect which can be neglected

$$
\overline{P_{i}} \approx \sum_{j=1}^{i} \overline{P_{e, j}}, \quad \overline{P_{B}}=\overline{P_{m}} \approx \sum_{i=1}^{m} \overline{P_{e, i}}
$$

i.e., the upper bound in (26) is asymptotically tight. Noting that the diversity order increases with the step number $i$, the smallest one being at step 1 , which results asymptotically in $\bar{P}_{e, 1} \gg$ $\bar{P}_{e, 2} \gg \ldots \gg \bar{P}_{e, m},(28)$ can be further approximated as

$$
\overline{P_{i}} \approx \overline{P_{e, 1}} \approx \overline{P_{B}} .
$$

Clearly, the first-step BER has the dominant effect, which agrees well with intuitive expectation based on the diversity order argument. The analysis above, however, gives a more detailed and precise picture. Note also that the approximation in (28) is more accurate than that in (29).

For convenience, Table I provides a summary of various error-rate definitions used in the paper.

\section{B. Relationship Between BLER and TBER}

After all the substreams have been detected, they are merged together into a single output stream. Let us define a TBER as BER in that output stream

$$
P_{e t}=\lim _{N \rightarrow \infty} \frac{N_{e}}{N}
$$

where $N_{e}$ and $N$ are the total number of bit errors and of the transmitted bits, respectively (the convergence of the limit is in probability). Since $N_{e}=\sum_{i=1}^{m} N_{e i}$, where $N_{e i}$ is the number of errors in the $i$ th stream, $P_{e t}$ can be expressed as

$$
P_{e t}=\frac{1}{m} \sum_{i=1}^{m} P_{u i}
$$

where we have used $P_{u i}=\lim _{N \rightarrow \infty}\left(m N_{e i} / N\right)$. While a step BER provides detailed characterization of the algorithm performance 
at each step, TBER represents an important performance measure of the system as a whole. One can upper and lower bound it using BLER derived above as follows. Define a block error as an event of having at least one error in the transmitted block of $m$ bits. Clearly, a block error means one to $m$ bit errors. Hence, the number of block and bit errors $\left(N_{B e}\right.$ and $\left.N_{e}\right)$ are related as $N_{B e} \leq N_{e} \leq m N_{B e}$, and the upper and lower bounds on $P_{e t}$ follow

$$
\frac{1}{m} P_{B} \leq P_{e t} \leq P_{B}
$$

which is similar to the relationship between symbol-error rates (SERs) and BERs [10]. While evaluation of $P_{\text {et }}$ requires precise characterization of the error-propagation effect, the bounds in (32) can be easily evaluated using the conditional BER only, which is much more simple. These bounds provide a reasonable estimate for $P_{e t}$ when $m$ is not too large. The upper bound in (32) also gives TBER, assuming $100 \%$ error propagation.

Similar expressions also hold true for average error rates

$$
\begin{aligned}
\overline{P_{e t}} & =\frac{1}{m} \sum_{i=1}^{m} \overline{P_{u i}} \\
\frac{1}{m} \overline{P_{B}} & \leq \overline{P_{e t}} \leq \overline{P_{B}} .
\end{aligned}
$$

Using (28), one obtains, for large average SNR

$$
\frac{1}{m} \sum_{i=1}^{m} \overline{P_{e, i}} \leq \overline{P_{e t}} \leq \sum_{i=1}^{m} \overline{P_{e, i}}
$$

Or, neglecting higher order terms

$$
\frac{1}{m} \overline{P_{e, 1}} \leq \overline{P_{e t}} \leq \overline{P_{e, 1}}
$$

Note that the expressions above hold for any modulation format.

\section{EXACT BER EXPRESSIONS FOR BPSK MODULATION}

In this section, we derive exact closed-form expressions for step BER with BPSK modulation. We use the normalized weights $\left|\mathbf{w}_{i}\right|^{2}=1$ (note that normalization does not affect SNR, and hence, BER), so that the weight matrix $\mathbf{W}=\left[\mathbf{w}_{1}, \mathbf{w}_{2}, \ldots, \mathbf{w}_{m}\right]$ is semi-unitary (it is unitary only when $m=n$ )

$$
\mathbf{W}^{+} \mathbf{W}=\mathbf{I}
$$

This normalization corresponds to $\mathbf{w}_{i}=\mathbf{P}_{i} \mathbf{h}_{i} /\left|\mathbf{P}_{i} \mathbf{h}_{i}\right|$. For simplicity, we begin with a $2 \times n$ system. In our analysis, we follow an approach similar to that of the decision-feedback multiuser detection BER analysis [11], [17].

\section{A. $2 \times n V$-BLAST BER}

The output signal at step 2 is

$$
\widehat{r_{2}}=\mathbf{w}_{2}^{+} \mathbf{h}_{2} s_{2}+\mathbf{w}_{2}^{+} \mathbf{h}_{1} \Delta s_{1}+\mathbf{w}_{2}^{+} \boldsymbol{\xi}
$$

where $\mathbf{w}_{2}=\mathbf{h}_{2} /\left|\mathbf{h}_{2}\right|$ and $\Delta s_{1}=s_{1}-\hat{s}_{1}$. Conditioned on $\mathbf{h}_{2}$ and $\Delta s_{1}$, the ISTI $z_{21}=\mathbf{w}_{2}^{+} \mathbf{h}_{1} \Delta s_{1}$ is Gaussian

$$
\left.z_{21}\right|_{\mathbf{h}_{2}, \Delta s_{1}} \sim C N\left(0,\left|\Delta s_{1}\right|^{2}\right)
$$

To see this, we first note that for given $\mathbf{h}_{2}$ and $\Delta s_{1}, z_{21}$ is a sum of Gaussian random variables, and, hence, is Gaussian. The conditional mean and variance of $z_{21}$ are

$$
\left\langle z_{21}\right\rangle=0 \quad\left\langle\left|z_{21}\right|^{2}\right\rangle=\mathbf{w}_{2}^{+}\left\langle\mathbf{h}_{1} \mathbf{h}_{1}^{+}\right\rangle \mathbf{w}_{2}\left|\Delta s_{1}\right|^{2}=\left|\Delta s_{1}\right|^{2}
$$

as $\left\langle\mathbf{h}_{1}\right\rangle=\mathbf{0}$ and $\left\langle\mathbf{h}_{1} \mathbf{h}_{1}^{+}\right\rangle=\mathbf{I}$. Since the conditional distribution does not depend on $\mathbf{h}_{2}$, the conditioning on $\mathbf{h}_{2}$ can be dropped

$$
\left.z_{21}\right|_{\Delta s_{1}} \sim C N\left(0,\left|\Delta s_{1}\right|^{2}\right)
$$

In the same way, one can see that the noise term in (38) is also Gaussian and independent of $\mathbf{h}_{2}$

$$
\mathbf{w}_{2}^{+} \boldsymbol{\xi} \sim C N\left(0, \sigma_{0}^{2}\right)
$$

and it is also independent of the other terms. The last two terms can be joined to get the "total noise" term, which includes ISTI, and, conditioned on $\Delta s_{1}$, its distribution is

$$
\mathbf{w}_{2}^{+} \mathbf{h}_{1} \Delta s_{1}+\mathbf{w}_{2}^{+} \boldsymbol{\xi} \sim C N\left(0, \sigma_{0}^{2}+\left|\Delta s_{1}\right|^{2}\right)
$$

Since instantaneous BER at each step are independent of each other and $\left|\Delta s_{1}\right|=0$ (no error at step 1) with probability (w.p.) $1-Q\left(\sqrt{2 \gamma_{1}}\right)$ and $\left|\Delta s_{1}\right|=2$ (error at step 1) w.p. $Q\left(\sqrt{2 \gamma_{1}}\right)$, for given $\mathbf{h}_{2}$, BER at step 2 can be immediately found from (38) as

$$
P_{u 2}\left(\mathbf{h}_{2}\right)=Q\left(\sqrt{2 \gamma_{2}}\right)\left(1-\bar{P}_{e 1}\right)+Q\left(\sqrt{\frac{2 \mathbf{h}_{2}^{+} \mathbf{h}_{2}}{\sigma_{0}^{2}+4}}\right) \bar{P}_{e 1}
$$

where $\bar{P}_{e 1}=\left\langle Q\left(\sqrt{2 \gamma_{1}}\right)\right\rangle_{\gamma_{1}}$ is the average BER at step 1, which is the same as the $(n-1)$-order MRC average BER given by [10]

$$
\begin{aligned}
\bar{P}_{e 1} & =\bar{P}_{(n-1)}^{\mathrm{MRC}}\left(\gamma_{0}\right) \\
& =\left[\frac{1-\mu}{2}\right]^{n-1} \sum_{k=0}^{n-2} C_{n-2+k}^{k}\left[\frac{1+\mu}{2}\right]^{k} \\
\mu & =\sqrt{\frac{\gamma_{0}}{1+\gamma_{0}}}
\end{aligned}
$$


where $C_{n}^{k}=n ! /(k !(n-k) !)$ are the binomial coefficients and, with the adopted normalization, $\gamma_{0}=1 / \sigma_{0}^{2}$ is the average SNR. $P_{u 2}\left(\mathbf{h}_{2}\right)$ is unconditional BER at step 2 averaged over $\mathbf{h}_{1}$ but not $\mathbf{h}_{2}$ (it is neither average nor instantaneous BER in traditional sense). Finally, the average unconditional BER at step 2 is

$$
\bar{P}_{u 2}=\left\langle P_{u 2}\left(\mathbf{h}_{2}\right)\right\rangle_{\mathbf{h}_{2}}=\bar{P}_{e 2}\left(1-\bar{P}_{e 1}\right)+\bar{P}_{21} \bar{P}_{e 1}
$$

where $\bar{P}_{e 2}=\left\langle Q\left(\sqrt{2 \gamma_{2}}\right)\right\rangle_{\gamma_{2}}$ is the conditional (on no error at the first step) average BER at step 2, which is the same as the $n$ th-order MRC average BER, $\bar{P}_{e 2}=\bar{P}_{(n)}^{\mathrm{MRC}}$, and

$$
\bar{P}_{21}=\left\langle Q\left(\sqrt{\frac{2 \mathbf{h}_{2}^{+} \mathbf{h}_{2}}{\sigma_{0}^{2}+4}}\right)\right\rangle_{\mathbf{h}_{2}}=\bar{P}_{(n)}^{\mathrm{MRC}}\left(\frac{1}{\sigma_{0}^{2}+4}\right)
$$

is the average probability of error propagation (the last equality in (47) follows from the observation that the "equivalent average SNR," which includes the propagating error as a part of the total noise, is $\left.1 /\left(\sigma_{0}^{2}+4\right)\right)$. The total average BER is found using (33)

$$
\overline{P_{e t}}=\frac{1}{2}\left(\overline{P_{e 1}}+\overline{P_{u 2}}\right) .
$$

In the case of a $2 \times 2$ system, the expressions become especially simple

$$
\begin{aligned}
& \bar{P}_{e 1}=\frac{1}{2}\left(1-\sqrt{\frac{\gamma_{0}}{1+\gamma_{0}}}\right) \\
& \bar{P}_{e 2}=\frac{1}{4}\left(1-\sqrt{\frac{\gamma_{0}}{1+\gamma_{0}}}\right)^{2}\left(2+\sqrt{\frac{\gamma_{0}}{1+\gamma_{0}}}\right) .
\end{aligned}
$$

Using these expressions and (33), the total BER can be found. For large average SNR $\sigma_{0}^{2} \ll 1$

$$
\begin{gathered}
\bar{P}_{21}=\bar{P}_{e 2}\left(\frac{1}{\sigma_{0}^{2}+4}\right) \approx \frac{1}{5} \\
\bar{P}_{e 2} \approx \frac{3}{4 \gamma_{0}^{2}} \\
\bar{P}_{e 1} \approx \frac{1}{4 \gamma_{0}}
\end{gathered}
$$

and (46) simplifies to

$$
\bar{P}_{u 2} \approx \bar{P}_{e 2}+\bar{P}_{21} \bar{P}_{e 1} \approx \frac{1}{5} \bar{P}_{e 1} \approx \frac{1}{20 \gamma_{0}} .
$$

Note that the second term dominates in (46), as it is the firstorder term (no diversity) while the first term is the second-order one (second-order diversity). Clearly, there is no diversity at the second step due to error propagation. The total average BER is

$$
\bar{P}_{e t} \approx \frac{1}{2} \bar{P}_{e 1} \approx \frac{1}{8 \gamma_{0}}
$$

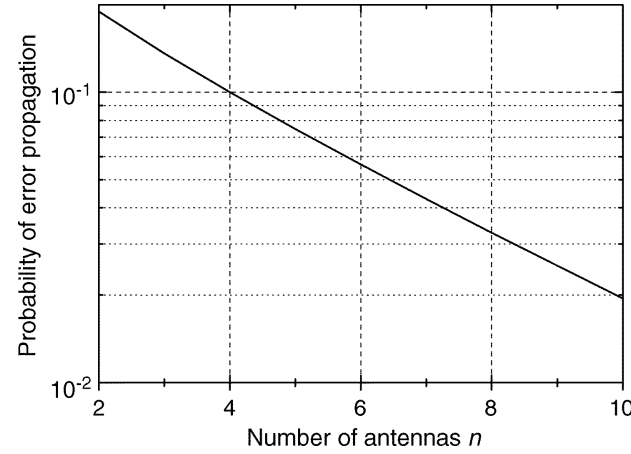

Fig. 1. Average probability of error propagation $\overline{P_{21}}$ for BPSK-modulated $2 \times$ $n$ V-BLAST.

The average BLER in this case is

$$
\bar{P}_{B}=\bar{P}_{e 2}\left(1-\bar{P}_{e 1}\right)+\bar{P}_{e 1}
$$

which can be approximated, for high average SNR, as

$$
\bar{P}_{B} \approx \bar{P}_{e 1} \approx \frac{1}{4 \gamma_{0}}
$$

We note that the approximation in (53) is the same as the lower bound in (34) and (36). Hence, the lower bound is asymptotically tight.

The high-SNR approximations above can also be extended to the $2 \times n$ case

$$
\begin{aligned}
& \bar{P}_{21} \approx \bar{P}_{(n)}^{\mathrm{MRC}}\left(\frac{1}{4}\right) \\
& \bar{P}_{e 2} \approx \frac{C_{2 n-1}^{n}}{\left(4 \gamma_{0}\right)^{n}} \\
& \bar{P}_{e 1} \approx \frac{C_{2 n-3}^{n-1}}{\left(4 \gamma_{0}\right)^{n-1}} \\
& \bar{P}_{u 2} \approx \bar{P}_{21} \bar{P}_{e 1} \\
& \bar{P}_{e t} \approx \frac{1}{2} \bar{P}_{e 1}\left(1+\bar{P}_{21}\right) \approx \frac{1}{2} \bar{P}_{e 1} \\
& \bar{P}_{B} \approx \bar{P}_{e 1} \approx \frac{C_{2 n-3}^{n-1}}{\left(4 \gamma_{0}\right)^{n-1}} .
\end{aligned}
$$

Note that the high-SNR approximation does not apply to $\bar{P}_{(n)}^{\mathrm{MRC}}(1 / 4)$, as the "effective" SNR for this term is $1 / 4$ (due to the propagating error from the first step).

Fig. 1 shows the average probability of error propagation $\bar{P}_{21}$ in (56) versus $n$. Clearly, the beneficial effect of increasing $n$ is not only to reduce $\bar{P}_{e 1}$ and $\bar{P}_{e 2}$ (by increasing the diversity order), but also to decrease the error propagation from the first step to the second. We attribute this to effective "beamforming" in the second step $\left(\mathbf{w}_{2}^{+}\right.$in (38)), which eliminates the propagating error when its angle of arrival (determined by $\mathbf{h}_{1}$ ) is not within the "main beam" of $\mathbf{w}_{2}^{+}$. As increasing $n$ will decrease 
the "beamwidth" [8], it will also decrease the probability of the propagating error to fall within the "main beam" of $\mathbf{w}_{2}^{+}$.

\section{B. $m \times n V$-BLAST BER}

Similarly to (38), the output signal (decision variable) at step $i$ is

$$
\widehat{r_{i}}=\mathbf{w}_{i}^{+} \mathbf{h}_{i} s_{i}+\mathbf{w}_{i}^{+} \sum_{k=1}^{i-1} \mathbf{h}_{k} \Delta s_{k}+\mathbf{w}_{i}^{+} \boldsymbol{\xi} .
$$

The ISTI term $z_{i}=\mathbf{w}_{i}^{+} \sum_{k=1}^{i-1} \mathbf{h}_{k} \Delta s_{k}$ is Gaussian conditioned on $\Delta \mathbf{S}_{i-1}=\left[\Delta s_{1}, \Delta s_{2}, \ldots, \Delta s_{i-1}\right]$

$$
\left.z_{i}\right|_{\Delta \mathbf{S}_{i-1}} \sim C N\left(0, \sigma_{z_{i}}^{2}\right)
$$

where

$$
\sigma_{z_{i}}^{2}=\mathbf{w}_{i}^{+} \sum_{k_{1}=1}^{i-1} \sum_{k_{2}=1}^{i-1}\left\langle\mathbf{h}_{k_{1}} \mathbf{h}_{k_{2}}^{+}\right\rangle \Delta s_{k_{1}} \Delta s_{k_{2}} \mathbf{w}_{i}=4\left|\mathbf{E}_{i-1}\right|^{2}
$$

and the expectation is over $\left\{\mathbf{h}_{1}, \mathbf{h}_{2}, \ldots, \mathbf{h}_{i-1}\right\} ; \mathbf{E}_{i-1}=$ $\left[e_{1}, e_{2}, \ldots, e_{i-1}\right]$ is the error vector, $e_{k}=1$ if there is an error at step $k$, and $e_{k}=0$ otherwise. The ISTI and noise terms in (60) are added up to the "total noise"

$$
\mathbf{w}_{i}^{+} \sum_{k=1}^{i-1} \mathbf{h}_{k} \Delta s_{k}+\mathbf{w}_{i}^{+} \boldsymbol{\xi} \sim C N\left(0, \sigma_{0}^{2}+4\left|\mathbf{E}_{i-1}\right|^{2}\right) .
$$

Let us consider conditional BER at step $i$ averaged over $\left\{\mathbf{h}_{1}, \mathbf{h}_{2}, \ldots, \mathbf{h}_{i-1}\right\}$

$$
P_{e, i \mid \mathbf{E}_{i-1}}=\operatorname{Pr}\left\{e_{i}=1 \mid \mathbf{E}_{i-1}\right\}=Q\left(\sqrt{\frac{2 \mathbf{h}_{i}^{+} \mathbf{P}_{i} \mathbf{h}_{i}}{\sigma_{0}^{2}+4\left|\mathbf{E}_{i-1}\right|^{2}}}\right) .
$$

$\operatorname{Pr}\left\{\mathbf{E}_{i-1}\right\}$ can be expressed as

$$
\operatorname{Pr}\left\{\mathbf{E}_{i-1}\right\}=\prod_{k=1}^{i-1} \operatorname{Pr}\left\{e_{k} \mid \mathbf{E}_{k-1}\right\}
$$

where

$$
\operatorname{Pr}\left\{e_{k} \mid \mathbf{E}_{k-1}\right\}= \begin{cases}P_{e, k \mid \mathbf{E}_{k-1}}, & e_{k}=1 \\ 1-P_{e, k \mid \mathbf{E}_{k-1}}, & e_{k}=0\end{cases}
$$

Let us define the following probability:

$$
P_{i}(\mathbf{H})=\sum_{\mathbf{E}_{i-1}} P_{e, i \mid \mathbf{E}_{i-1}} \operatorname{Pr}\left\{\mathbf{E}_{i-1}\right\}
$$

where the summation is over all possible $\mathbf{E}_{i-1}$. Note that this is neither instantaneous nor average BER at step $i$, as some terms are averaged over some of $\mathbf{h}_{k}, k=1, \ldots, i-1$, and other terms are not [similar to (44)]. Using (67) and the independence of

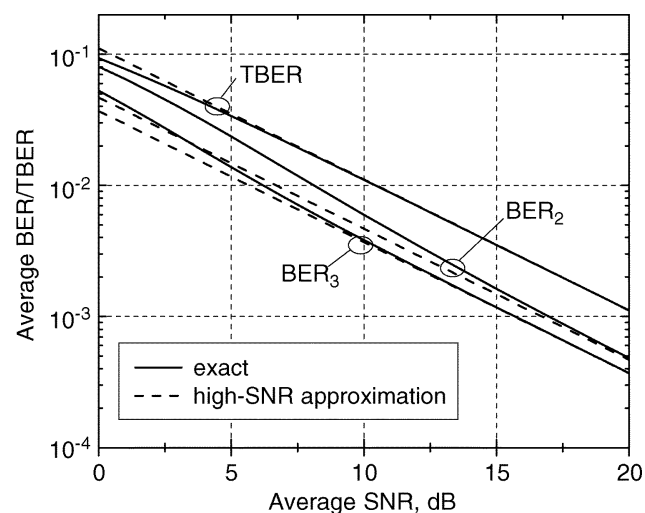

Fig. 2. Average BER/TBER of $3 \times 3$ BPSK-modulated V-BLAST. Exact versus high-SNR approximation.

SNR at each step, it is straightforward to obtain average unconditional BER

$$
\bar{P}_{u, i}=\left\langle P_{u, i}(\mathbf{H})\right\rangle_{\mathbf{H}}=\sum_{\mathbf{E}_{i-1}} \bar{P}_{e, i \mid \mathbf{E}_{i-1}} \bar{P}\left[\mathbf{E}_{i-1}\right]
$$

where

$$
\begin{aligned}
\bar{P}_{e, i \mid \mathbf{E}_{i-1}} & =\bar{P}_{(n-m+i)}^{\mathrm{MRC}}\left(\frac{1}{\sigma_{0}^{2}+4\left|\mathbf{E}_{i-1}\right|^{2}}\right) \\
\bar{P}\left[\mathbf{E}_{i-1}\right] & =\prod_{k=1}^{i-1}\left\langle\operatorname{Pr}\left\{e_{k} \mid \mathbf{E}_{k-1}\right\}\right\rangle
\end{aligned}
$$

and $\left\langle\operatorname{Pr}\left\{e_{k} \mid \mathbf{E}_{k-1}\right\}\right\rangle$ are found using the expectation of (66) over appropriate entries of $\mathbf{H}$. Similar to (47), the expression for $\bar{P}_{e, i} \mid \mathbf{E}_{i-1}$ follows from that for the traditional MRC BPSK average BER by observing that the "equivalent average SNR" in (64) is $1 /\left(\sigma_{0}^{2}+4\left|\mathbf{E}_{i-1}\right|^{2}\right)$. Using (33) and (68), the total average BER can be easily obtained

$$
\overline{P_{e t}}=\frac{1}{m} \sum_{i=1}^{m} \sum_{\mathbf{E}_{i-1}} \bar{P}_{e, i \mid \mathbf{E}_{i-1}} \bar{P}\left[\mathbf{E}_{i-1}\right] .
$$

Similar to the $2 \times n$ case, high-SNR approximations can be developed from the expressions above. The first-step BER still has a dominant effect on the overall performance, i.e., the diversity order at each step and also in the total average BER is the same as in the first step, $(n-m+1)$. Thus, the dominant contribution to $\bar{P}_{u, i}$ in (68) is coming from such error vectors that have an error at the first step, $\tilde{\mathbf{E}}_{i-1}=\left[1, e_{2}, \ldots, e_{i-1}\right]$, and the summation in (68) has to be performed only over such vectors

$$
\bar{P}_{u, i} \approx \sum_{\left[e_{2}, \ldots, e_{i-1}\right]} \bar{P}_{e, i \mid \tilde{\mathbf{E}}_{i-1}} \bar{P}\left[\tilde{\mathbf{E}}_{i-1}\right]
$$

where $\bar{P}_{e, i \mid \tilde{\mathbf{E}}_{i-1}}$ can be approximated as

$$
\bar{P}_{e, i \mid \tilde{\mathbf{E}}_{i-1}} \approx \bar{P}_{(n-m+i)}^{\mathrm{MRC}}\left(\frac{1}{4\left|\tilde{\mathbf{E}}_{i-1}\right|^{2}}\right) .
$$


TABLE II

AVERAGE BER COEFFICIENTS IN (73) AND (75) FOR $n \times n$ SYSTEM.

\begin{tabular}{|c|c|c|c|c|c|c|c|c|c|}
\hline$i(n)$ & 2 & 3 & 4 & 5 & 6 & 7 & 8 & 9 & 10 \\
\hline$a_{i}$ & 0.187 & 0.148 & 0.122 & 0.103 & 0.089 & 0.078 & 0.068 & 0.061 & 0.055 \\
\hline$a_{t}$ & 0.594 & 0.445 & 0.364 & 0.311 & 0.275 & 0.247 & 0.224 & 0.206 & 0.191 \\
\hline
\end{tabular}

Since each term in (71) includes $\bar{P}_{e 1}$ as a co-factor, it can be factored out, and hence, (71) can be presented in the following form:

$$
\begin{aligned}
\bar{P}_{u, i} & \approx a_{i} \bar{P}_{e 1} \\
a_{i} & =\sum_{\left[e_{2}, \ldots, e_{i-1}\right]} \bar{P}_{e, i \mid \tilde{\mathbf{E}}_{i-1}} \prod_{k=2}^{i-1}\left\langle\operatorname{Pr}\left(e_{k} \mid \tilde{\mathbf{E}}_{k-1}\right)\right\rangle
\end{aligned}
$$

where $\left\langle\operatorname{Pr}\left(e_{k} \mid \tilde{\mathbf{E}}_{k-1}\right)\right\rangle$ is found using (66) and the high-SNR approximation in (72)

$$
\left\langle\operatorname{Pr}\left\{e_{k} \mid \tilde{\mathbf{E}}_{k-1}\right\}\right\rangle= \begin{cases}\bar{P}_{(n-m+k)}^{\mathrm{MRC}}\left(\frac{1}{4\left|\tilde{\mathbf{E}}_{k-1}\right|^{2}}\right), & e_{k}=1 \\ 1-\bar{P}_{(n-m+k)}^{\mathrm{MRC}}\left(\frac{1}{4\left|\tilde{\mathbf{E}}_{k-1}\right|^{2}}\right), & e_{k}=0\end{cases}
$$

and $a_{1}=1, a_{2}=\bar{P}_{21}$, and $\bar{P}_{e 1} \approx C_{2(n-m)+1}^{n-m+1} /\left(4 \gamma_{0}\right)^{n-m+1}$. Finally, the total average BER is expressed as

$$
\overline{P_{e t}} \approx a_{t} \bar{P}_{e 1}, \quad a_{t}=\frac{1}{m} \sum_{i=1}^{m} a_{i}
$$

Note that $a_{i}$ are constants at high SNR. Fig. 2 shows that the approximations in (73)-(75) are indeed accurate, even for moderately large SNR (especially for the total average BER). Table II gives the coefficients $a_{i}$ and $a_{t}$ for the $n \times n$ system. Note that $a_{i}$ with $i \leq n$ is independent of $n$. In fact, it depends only on $(n-m)$, but not $n$ and $m$ individually, e.g., $a_{2}$ is the same for all $n \times n$ systems with $n \geq 2$. $a_{t}$ represents the total average BER as the percentage of the first-step average BER. Clearly, it decreases with $n$.

It should also be noted that the BER performance of uncoded D-BLAST can also be evaluated using the expressions above. Specifically, by observing that the antenna cycling in D-BLAST [1], [2] does not affect $\overline{P_{e t}}$ (since all the detected substreams are finally merged into the single output stream), all the expressions above for $\overline{P_{e t}}$ apply directly to D-BLAST as well. However, due to the antenna cycling, all the average substream error rates $\bar{P}_{u, i(\mathrm{D}-\mathrm{BLAST})}$, where index $i$ is associated now with the substream rather than the detection step, will be the same (since each particular substream is detected periodically at each step from 1 to $m$ ), and hence, equal to the average total error rate $\bar{P}_{u, i(\mathrm{D}-\mathrm{BLAST})}=\overline{P_{e t}}$. Thus, D-BLAST does not offer any BER advantage over V-BLAST in terms of the total error rate. The advantage is coming from the fact that all substreams perform equally well.

It should be pointed out that a $\mathrm{QR}$ decomposition of the channel matrix $\mathbf{H}=\mathbf{Q R}$, where $\mathbf{Q}$ is semiunitary, $\mathbf{Q}^{+} \mathbf{Q}=\mathbf{I}$, and $\mathbf{R}$ is lower triangular, can be used for the interference-nulling procedure instead of the projections with signif- icant reduction in computational complexity of the algorithm [12], [13], [20]. These two algorithms are identical in terms of decision variables, and hence, in terms of the performance [19], so that all the results here also apply to the QR-based V-BLAST. The identical performance of these two algorithms can also be seen directly from (37) as the weight matrix W is semiunitary, and $\mathbf{W}^{+} \mathbf{H}=\mathbf{T}$, where $\mathbf{T}$ is lower triangular (since $\mathbf{w}_{i}^{+} \mathbf{h}_{j}=0 \forall i<j$ ), is also a QR decomposition of $\mathbf{H}$. Since $\mathrm{QR}$ decomposition is unique [7], $\mathbf{W}=\mathbf{Q}, \mathbf{R}=\mathbf{T}$, and the two algorithms are identical. For an i.i.d. Rayleigh channel, the entries of $\mathbf{H}$ are i.i.d. complex Gaussian, $h_{i j} \sim \mathcal{C N}(0,1)$, and the $\mathrm{QR}$ decomposition above has the following properties [7]:2 1) $r_{i i} \sim \chi_{2(n-m+i)}^{2}$;2) $r_{i j} \sim \mathcal{C N}(0,1), i>j$; 3) all the elements of $\mathbf{R}$ are independent of each other; 4) the elements of $\mathbf{Q}$ and $\mathbf{R}$ are independently distributed and, hence, the noise $\boldsymbol{\xi}^{\prime}=\mathrm{Q}^{+} \boldsymbol{\xi}$ is independent of the signal term $\mathbf{Q}^{+} \mathbf{H}=\mathbf{R s}$; furthermore, $\boldsymbol{\xi}^{\prime} \sim \boldsymbol{\xi} \sim \mathcal{C N}\left(\mathbf{0}, \sigma_{0}^{2} \mathbf{I}\right)$. These properties are identical to the results obtained above using the step-by-step analysis of the algorithm, and hence, indirectly validate our earlier results. Property 3 also confirms that $P_{s i}$ in (4), and hence, the instantaneous SNRs at different steps are independent of each other, which facilitates the average error-rate analysis.

\section{Monte Carlo Simulations}

In order to verify the analytical results above, extensive MC simulations have been undertaken. Specifically, the Rayleigh i.i.d. fading channel and BPSK modulation demodulated coherently have been used. First, the instantaneous BER expressions have been validated. No statistically significant difference between analytical and MC results have been found for conditional (without error propagation) and unconditional (with error propagation) BER. Secondly, the average BER expressions have been extensively validated. Some of the results are shown in Figs. 3-6.

Figs. 3 and 4 show MC simulated average BER/BLER/TBER and the exact analytical results in Section V for $2 \times 2$ and $2 \times 3$ systems. Good agreement is obvious. First-step BER dominates the BLER for high SNR ( $>5 \mathrm{~dB})$. Note that the error propagation has significant effect on the second-step BER, as comparison to the "no error propagation" BER demonstrates (as (52) indicates, the error propagation results in the second-step diversity order being equal to one rather than two).

Fig. 5 compares the exact and approximate average BLER/ TBER expressions (33) and (53)-(55) of a $2 \times 2$ system. Clearly, the average BLER is well approximated by the first-step average BER for $\gamma_{0} \geq 5 \mathrm{~dB}$. The accuracy of the TBER approximation is a bit worse but still acceptable. The small inaccuracy is due to the fact that (53) ignores the error propagation, which demon-

\footnotetext{
${ }^{2}$ An elegant derivation of these properties, which is based on the Householder transformation, can be found in [20].
} 


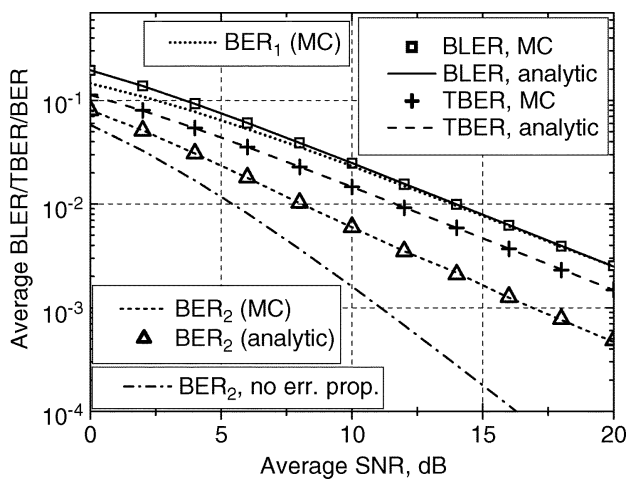

Fig. 3. Average BLER/TBER/BER of $2 \times 2$ BPSK-modulated V-BLAST.

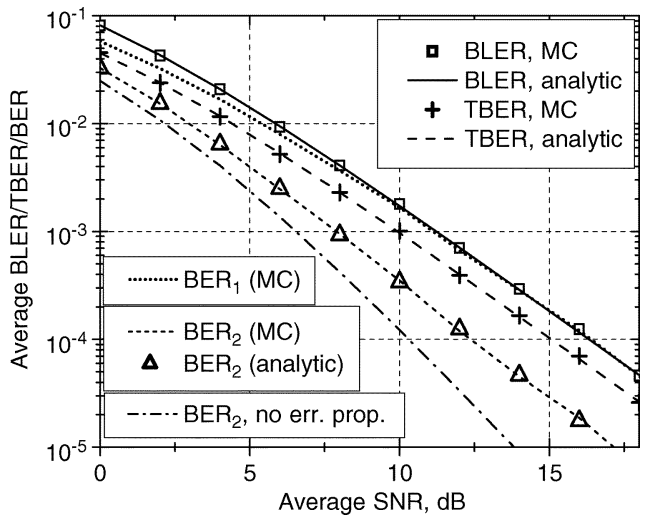

Fig. 4. Average BLER/TBER/BER of $2 \times 3$ BPSK-modulated V-BLAST.

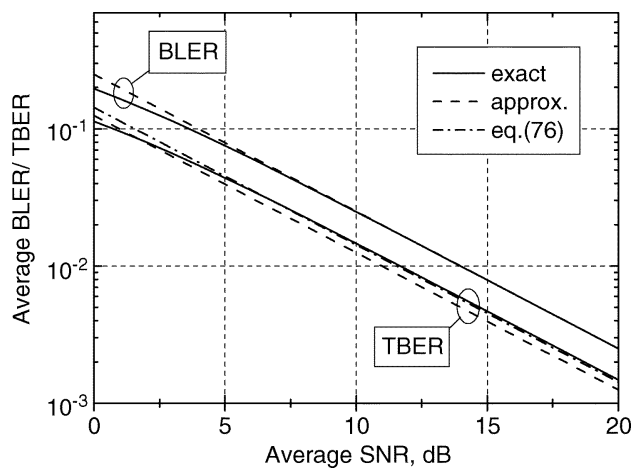

Fig. 5. Average BLER/TBER of $2 \times 2$ BPSK-modulated V-BLAST. Exact and approximate.

strates that the effect of error propagation on the total average BER is indeed small. Including it results in a better approximation

$$
\bar{P}_{e t} \approx \frac{\bar{P}_{e 1}}{2}\left(1+\bar{P}_{21}\right) \approx \frac{1}{7 \gamma_{0}} .
$$

Comparing (76) with (53), one concludes that the effect of error propagation on the average total BER is to increase it by about $20 \%$, which is indeed small (contrary to an existing viewpoint that the error propagation has a dramatically negative effect on BLAST BER). On the contrary, the second-step average BER is affected by the error propagation in a dramatic way:

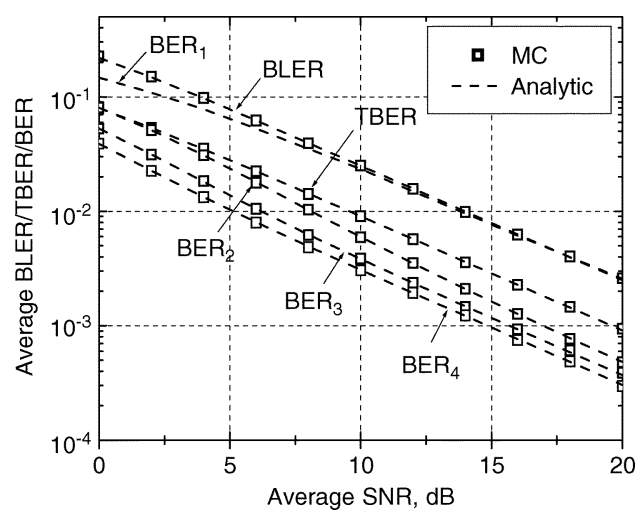

Fig. 6. Average BLER/TBER/BER of $4 \times 4$ BPSK-modulated V-BLAST.

the diversity order decreases from two without error propagation to one with it (see Figs. 3 and 4). Fortunately, since the first-step BER is dominant, this does not significantly affect the total BER.

Fig. 6 gives the average step BER of a $4 \times 4$ system along with the average BLER/TBER. Based on these numerical results, one observes that the average step BER, despite the error propagation, decreases with step number

$$
\bar{P}_{u(i+1)}<\bar{P}_{u i} .
$$

According to the analysis above, we attribute this to the fact that: 1$)$ the probability of error propagation is less than $1(\approx 1 / 5$ for a $2 \times 2$ system); and 2) the conditional BER at higher steps has higher diversity order (see (47), (51), and (52) for the case of a $2 \times 2$ system). Clearly, the average step BER of an $n \times n$ system can be well approximated by (73), where $a_{i+1}<a_{i}$ (see also Table II). Based on numerical evidence, we conjecture that $\lim a_{i}=0$. Similar approximation also applies to BLER and $\stackrel{i \rightarrow \infty}{\text { TBER [ }}$ [see (59) and (75)]. Additionally, one observes that (29) is indeed an accurate approximation, and that the lower bounds in (34) and (36) are tight for high SNR, $\bar{P}_{e t} \approx \bar{P}_{B} / m \approx \bar{P}_{e 1} / m$. Based on this, we conjecture that $\lim _{n \rightarrow \infty} \bar{P}_{\text {et }}=0$ for an $n \times n$ system, which constitutes the autocoding effect [21] in the unordered V-BLAST system. Comparing Fig. 6 with Fig. 3, one concludes that the average BER at given step does not depend on whether higher steps are present or not (for example, $\overline{P_{e 1}}$ is the same for $2 \times 2,3 \times 3$, and $4 \times 4$ systems), which also follows from (68), and is especially obvious for the high-SNR approximation in (73). This conclusion is not entirely trivial, as step 1 processing implies projecting out the subspace spanned by $\left[\mathbf{h}_{2}, \mathbf{h}_{3}, \ldots, \mathbf{h}_{m}\right]$ and, hence, depends on the presence of higher order steps.

\section{CONCLUSIONS}

Closed-form analytical performance evaluation of the V-BLAST algorithm has been presented in this paper. While the assumption of no ordering limits the results obtained, it also allows for such an evaluation to be performed, providing insight which is not available using numerical techniques only (i.e., MC simulations). It was demonstrated that the optimum (MRC) weights, which also include the projections, are orthogonal to 
each other and, hence, the after-combining noise is independent at each step, which simplifies the analysis significantly and results in closed-form expressions for the instantaneous BER at each step. The instantaneous SNR at each step are also independent of each other for a Rayleigh i.i.d. channel, and, additionally, the ISTI is Gaussian. Hence, the average BER expressions can be obtained in a straightforward way (using, for example, the techniques developed for multiuser detection analysis). In the case of BPSK modulation and high SNR, the expressions are especially simple. The error propagation has a dramatic effect on higher step BER, resulting in the diversity order being equal to $(n-m+1)$ at each step, but it has only a minor effect on the total average BER (about $20 \%$ increase for a $2 \times 2$ BPSK-modulated system at high SNR). The average step BER is lower for higher steps (since the probability of error propagation is less than one). As expected, the average BLER and total error rate are dominated by the first-step BER.

\section{APPENDIX}

Consider the after-projection noise vector

$$
\begin{aligned}
\boldsymbol{\xi}_{(i)} & =\mathbf{P}_{i} \boldsymbol{\xi} \\
\mathbf{P}_{i} & =\mathbf{I}-\mathbf{H}_{i}^{+}\left(\mathbf{H}_{i} \mathbf{H}_{i}^{+}\right)^{-1} \mathbf{H}_{i} .
\end{aligned}
$$

Since the correlation matrix of the original noise vector is

$$
\left\langle\xi \xi^{+}\right\rangle=\sigma_{0}^{2} \mathbf{I}
$$

where the expectation is over the noise, the correlation matrix of the projected noise is

$$
\mathbf{C}_{\xi}=\left\langle\boldsymbol{\xi}_{(i)} \boldsymbol{\xi}_{(i)}^{+}\right\rangle=\sigma_{0}^{2} \mathbf{P}_{i} \mathbf{P}_{i}^{+}=\sigma_{0}^{2} \mathbf{P}_{i}
$$

where we used the following property of the projection matrix: $\mathbf{P}_{i}=\mathbf{P}_{i}^{+}=\mathbf{P}_{i} \mathbf{P}_{i}^{+}$(the first equality can be easily verified using (A1); the second one follows from the fact that the second projection is always redundant). Clearly, the noise is correlated. Let us consider the MRC for the correlated-noise case. To simplify the notations, we further drop index $i$. The output signal of the combiner is

$$
r_{\text {out }}=\mathbf{w}^{+} \mathbf{y} s+\mathbf{w}^{+} \boldsymbol{\xi}
$$

where $\mathbf{w}^{+}$is the weight vector, and $\mathbf{y} s=\mathbf{P h} s$ is the afterprojection signal; for simplicity, we further assume that $|s|^{2}=1$ (this does not affect the weight vector). The output SNR (to be maximized) is

$$
\gamma=\frac{P_{s}}{P_{\xi}}=\frac{\mathbf{w}^{+} \mathbf{C}_{s} \mathbf{w}}{\mathbf{w}^{+} \mathbf{C}_{\xi} \mathbf{w}}=\frac{\mathbf{w}^{+} \mathbf{P h h}^{+} \mathbf{P} \mathbf{w}}{\mathbf{w}^{+} \mathbf{P w}}
$$

where $P_{s}$ and $P_{\xi}$ are the signal and noise powers at the combiner output, and $\mathbf{C}_{s}=\mathbf{y} \mathbf{y}^{+}$is the signal instantaneous correlation matrix. The weight vector $\mathrm{w}^{+}$that maximizes (A5) can be found by using the Cauchy-Schwartz inequality [14]

$$
\gamma=\frac{\left|\tilde{\mathbf{h}}^{+} \tilde{\mathbf{w}}\right|^{2}}{\sigma_{0}^{2}|\tilde{\mathbf{w}}|^{2}} \leq \frac{|\tilde{\mathbf{h}}|^{2}}{\sigma_{0}^{2}}=\frac{|\mathbf{P h}|^{2}}{\sigma_{0}^{2}}
$$

where $\tilde{\mathbf{w}}=\mathbf{P w}, \tilde{\mathbf{h}}=\mathbf{P h}$. The equality in (A6) is achieved with

$$
\tilde{\mathbf{w}}=\tilde{\mathbf{h}} \text {. }
$$

This gives the generic form of $\mathbf{w}$ (up to a scalar factor) achieving the maximum

$$
\mathbf{w}=\mathbf{P h}+\Delta \mathbf{w}
$$

where $\mathbf{P} \Delta \mathbf{w}=\mathbf{0}$. We choose $\Delta \mathbf{w}=\mathbf{0}$, which gives

$$
\mathbf{w}=\mathbf{P h} .
$$

Note that one may also choose $\mathbf{w}=\mathbf{h}$, i.e., the projection does not affect the MRC weights and, despite the noise correlation due to the projection, the classical MRC solution still applies. The maximum output SNR is

$$
\gamma=\frac{|\mathbf{P h}|^{2}}{\sigma_{0}^{2}}=\frac{\mathbf{y}^{+} \mathbf{y}}{\sigma_{0}^{2}} .
$$

Remarkably, the output SNR is not affected by the noise correlation and is the same as if the noise were i.i.d. in each branch (after projection). We attribute it to the special structure of the projection matrix (A1). (A10) clearly indicates that the analysis in terms of the total signal power $\mathbf{y}^{+} \mathbf{y}$ is equivalent (up to a constant) to the analysis in terms of the SNR $\gamma$ if the MRC is used after the orthogonal projection.

\section{ACKNOWLEDGMENT}

The authors would like to thank H. Boelcskei, V. Kostina, the anonymous reviewers, and the editor for constructive comments and discussions.

\section{REFERENCES}

[1] G. J. Foschini et al., "Analysis and performance of some basic spacetime architectures," IEEE J. Sel. Areas Commun., vol. 21, no. 3, pp. 281-320, Apr. 2003

[2] G. J Foschini, "Layered space-time architecture for wireless communication in a fading environment when using multiple antennas," Bell Lab. Tech. J., vol. 1, no. 2, pp. 41-59, 1996.

[3] G. J. Foschini et al., "Simplified processing for high spectral efficiency wireless communication employing multi-element arrays," IEEE J. Sel. Areas Commun., vol. 17, no. 11, pp. 1841-1852, Nov. 1999.

[4] S. Loyka, "V-BLAST outage probability: Analytical analysis," in Proc. IEEE Veh. Technol. Conf., Vancouver, BC, Canada, Sep. 2002, pp. 24-28.

[5] S. Loyka and F. Gagnon, "Performance analysis of the V-BLAST algorithm: An analytical approach," IEEE Trans. Wireless Commun., vol. 3 , no. 4, pp. 1326-1337, Jul. 2004 
[6] - "Analytical framework for outage and BER analysis of the V-BLAST algorithm," in Proc. Int. Zurich Sem. Commun., Zurich, Switzerland, Feb. 2004, pp. 120-123.

[7] T. W. Anderson, An Introduction to Multivariate Analysis, 3 ed. New York: Wiley, 2003.

[8] H. L. Van Trees, Optimum Array Processing. New York: Wiley, 2002.

[9] W. C. Jakes, Jr., Microwave Mobile Communication. New York: Wiley, 1974.

[10] J. G. Proakis, Digital Communications. Boston: McGraw-Hill, 2001.

[11] S. Verdu, Multiuser Detection. Cambridge, U.K.: Cambridge Univ. Press, 1998.

[12] D. Wubben et al., "Efficient algorithm for decoding layered space-time codes," Electron. Lett., vol. 37, no. 22, pp. 1348-1350, Oct. 2001.

[13] J. Choi, "A bi-directional zero-forcing BLAST receiver," IEEE Trans. Signal Process., vol. 52, no. 9, pp. 2670-2673, Sep. 2004.

[14] Anonymous Reviewer, private communication.

[15] M. E. Austin, "Decision-feedback equalization for digital communication over dispersive channels," MIT Res. Lab. Electron., Cambridge, MA, Tech. Rep. 461, Aug. 1967.

[16] A. Duel-Hallen, "Decorrelating decision-feedback multiuser detector for synchronous code-division multiple access channel," IEEE Trans. Commun., vol. 41, no. 2, pp. 285-290, Feb. 1993.

[17] M. K. Varanasi, "Decision feedback multiuser detection: A systematic approach," IEEE Trans. Inf. Theory, vol. 45, no. 1, pp. 219-240, Jan. 1999.

[18] J. E. Smee and N. C. Beaulieu, "Error-rate evaluation of linear equalization and decision feedback equalization with error propagation," IEEE Trans. Commun., vol. 46, no. 5, pp. 656-665, May 1998.

[19] G. Ginis and J. M. Cioffi, "On the relation between V-BLAST and the GDFE," IEEE Commun. Lett., vol. 5, no. 9, pp. 364-366, Sep. 2001.

[20] T. L. Marzetta, "The QR factorization, and its role in a new high capacity, layered space-time architecture for wireless communication," Bell Labs Tech. Memo. BL0112170-960620-13TM, Jun. 1996.

[21] B. M. Hochwald and T. L. Marzetta, "Space-time autocoding," IEEE Trans. Inf. Theory, vol. 47, no. 7, pp. 2761-2781, Nov. 2001.

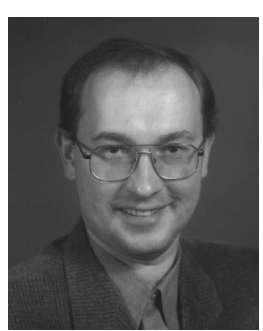

Sergey Loyka (M'96-SM'04) was born in Minsk, Belarus. He received the Ph.D. degree in radio engineering from the Belorussian State University of Informatics and Radioelectronics, Minsk, Belarus, in 1995, and the M.S. degree with honors from Minsk Radioengineering Institute, Minsk, Belarus, in 1992.

Since 2001, he has been a Faculty Member with the School of Information Technology and Engineering (SITE), University of Ottawa, Ottawa, ON, Canada. Prior to that, he was a Research Fellow with the Laboratory of Communications and Integrated Microelec- tronics (LACIME), Ecole de Technologie Superieure, Montreal, QC, Canada; a Senior Scientist with the Electromagnetic Compatibility Laboratory, Belorussian State University of Informatics and Radioelectronics, Minsk, Belarus; and an Invited Scientist at the Laboratory of Electromagnetism and Acoustic, Swiss Federal Institute of Technology, Lausanne, Switzerland. His research areas include wireless communications, MIMO systems and smart antennas, RF system modeling and simulation, and electromagnetic compatibility, in which he has published extensively.

Dr. Loyka is a Technical Program Committee member of several IEEE conferences and a reviewer for numerous IEEE and IEE periodicals and conferences. He received a number of awards from the URSI, the IEEE, the Swiss, Belarus and former USSR governments, and the Soros Foundation.

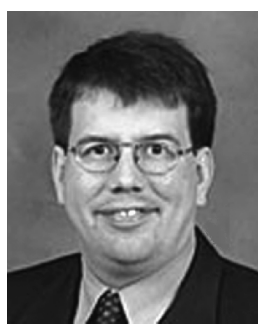

Francois Gagnon (''87-M'87-SM'99) was born in Québec City, QC, Canada. He received the B.Eng. and $\mathrm{Ph} . \mathrm{D}$. degrees in electrical engineering from École Polytechnique de Montréal, Montréal, QC, Canada.

Since 1991, he has been a Professor with the Department of Electrical Engineering, École de Technologie Supérieure, Montréal, QC, Canada. He chaired the department from 1999 to 2001, and is now the holder of the Ultra Electronics (TCS) Chair in Wireless Communication at the same university. His research interest covers wireless high-speed communications, modulation, coding, high-speed DSP implementations, and military point-to-point communications. He has been very involved in the creation of the new generation of high-capacity line-of-sight military radios offered by the Canadian Marconi Corporation, which is now Ultra Electronics Tactical Communication Systems. The company has received, for this product, a "Coin of Excellence" from the U.S. Army for performance and reliability. He has also been involved in defining programmable radios with Harris, Nortel, and Bell. He has written more than 45 scientific papers, holds one patent, and reviews papers for three IEEE journals. 